\title{
An Exploration of the Playing Style of Zheng Music Bamboo Rhyme
}

\author{
Bing $\mathrm{Hu}$ \\ School of Art and Design \\ Xi'an University of Technology \\ Xi'an, China
}

\begin{abstract}
Bamboo Rhyme" is a Zheng piece created by Professor Wei Jun of Xi'an Conservatory of Music, which is compact in composition, concise in technique and rich in scholarly flavor. Its appreciation of bamboo's noble character and the deep friendship with classmates is placed the thick affection of composer. The purpose of this paper is to elaborate on the playing style of this song through the careful analysis of "Bamboo Rhyme", so as to provide the player with some ideas and methods for reference.
\end{abstract}

Keywords-Zheng song; "bamboo rhyme"; playing style

\section{INTRODUCTION}

The song "Bamboo Rhyme" describes the shape and charm of bamboo, placing composers' compliments on the noble character of bamboo and affection for his classmates. This paper makes artistic analysis on "Bamboo Rhyme" from the aspects of music score and playing, trying to study the music through the features of structure, playing skills, playing art and aesthetics, in order to help the player better grasp the music direction and venation when playing, comprehend the spirit and temperament of the music, and at

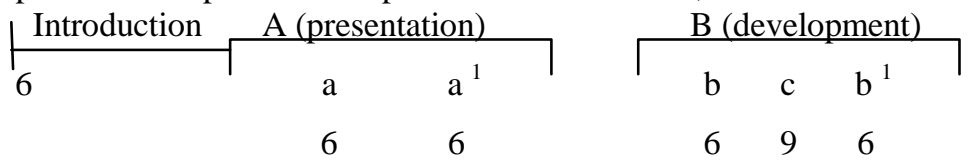

the same time broaden the research perspective of the performer to other Zheng music works. It is of theoretical and practical significance for studying the playing style of Zheng and the Chinese traditional aesthetic concept of "rhythmic vitality".

\section{ThE STRUCTURAL CHARACTERISTICS OF "BAMBOO RHYME"}

"Bamboo Rhyme" was created in 2004. On the thinking and formation of the song creation, the composer recalls that: "My classmate Yaoru knows rhyming, and is good at poetry. He can write characters well, of which Zhuanzi is the most superb. Therefore, I make "bamboo rhyme" as a gift to him to encourage each other." The accumulation of student life and years of friendship make the composer generate feeling and write this song in order to express the friendship of the best friend. The structure of this song is "reproducible single three", a complete work with symmetrical structure, as shown in "Fig. 1":

\section{A. Analysis of musical form of "Bamboo Rhyme"}

It consists of introduction, presentation $\mathrm{A}$, development $\mathrm{B}$, reappearance $\mathrm{A} 1$ and so on, with regular structure. The $=40$, which paves the way for the color of the whole song in quiet and calm temperament and guide the entire song. introduction includes 6 sections in total, with the speed of:

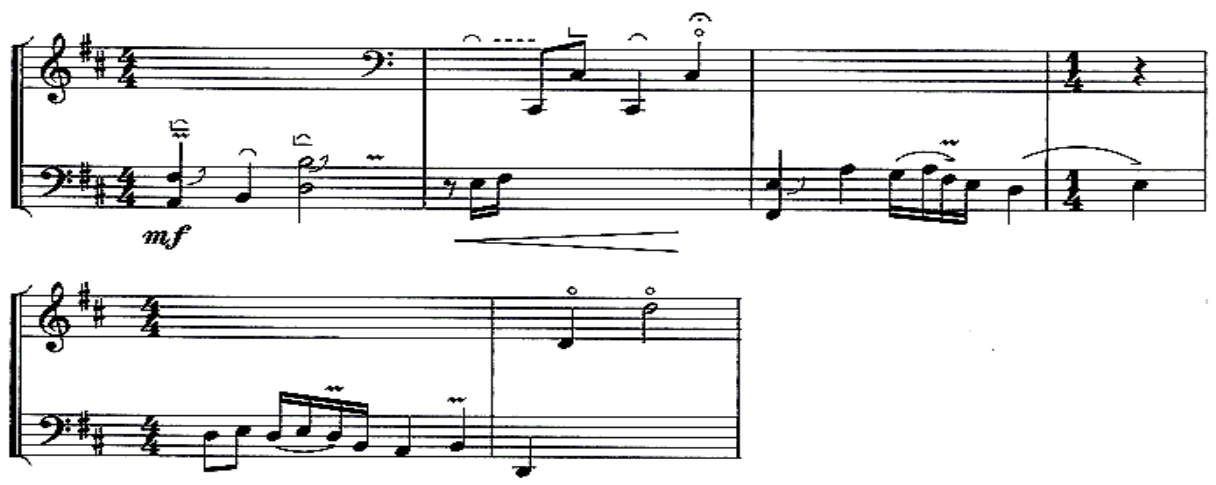

Fig. 1. Introduction section. 
Followed by the introduction is the segment A of presentation section. Presentation section is close parallel double sentence segment, and two phrases each include 6 sections, with a strong sense of balance. The two phrases are almost identical, and there is a repeated theme throughout the whole song. As "Fig. 2" shows:
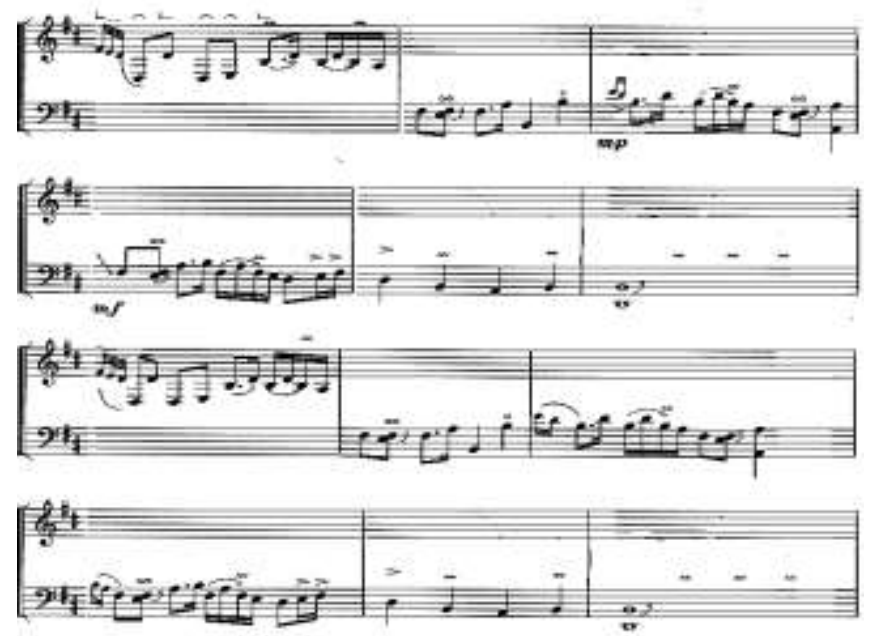

Fig. 2. Music theme of segment A.

Segment B is the development part of the whole song as the middle part of the mix, where there are both the expansion of old material and contrast of new material. material of Part A. Part b can be seen as a variation of the second half of segment A, to further reveal and emphasize the theme. As shown in the "Fig. 3":

Part b contains six sections, elaborated with overtones, and the old material is used to further explore the music

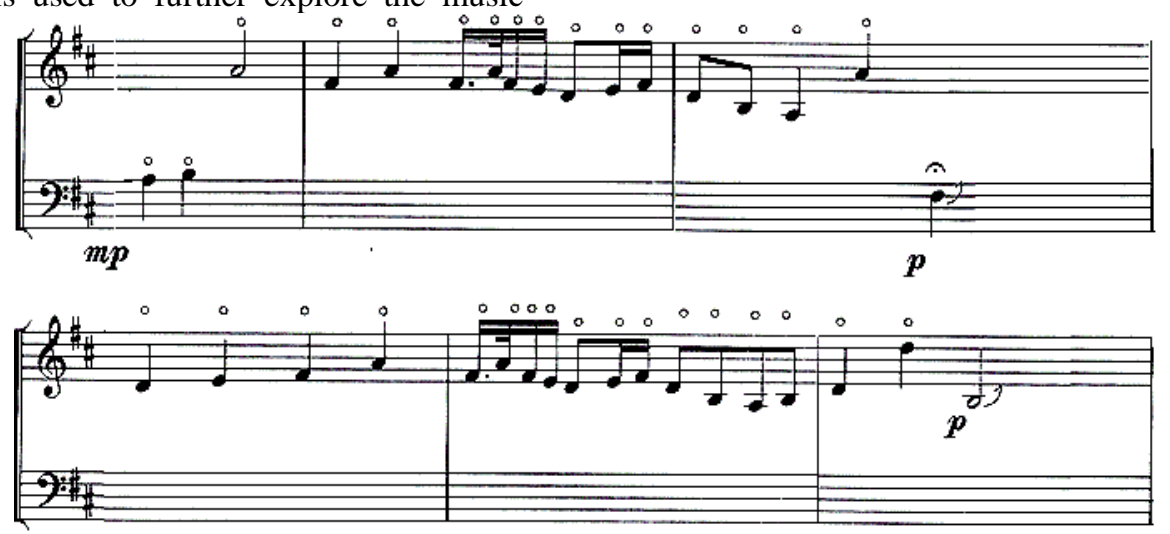

Fig. 3. Segment B, part b.

Part c is led by a new piece of material, showing positive emotion and musical progression. This segment includes 9 sections, with new material joining in, as well as the old material from introduction and segment A. As shown in "Fig. 4": 

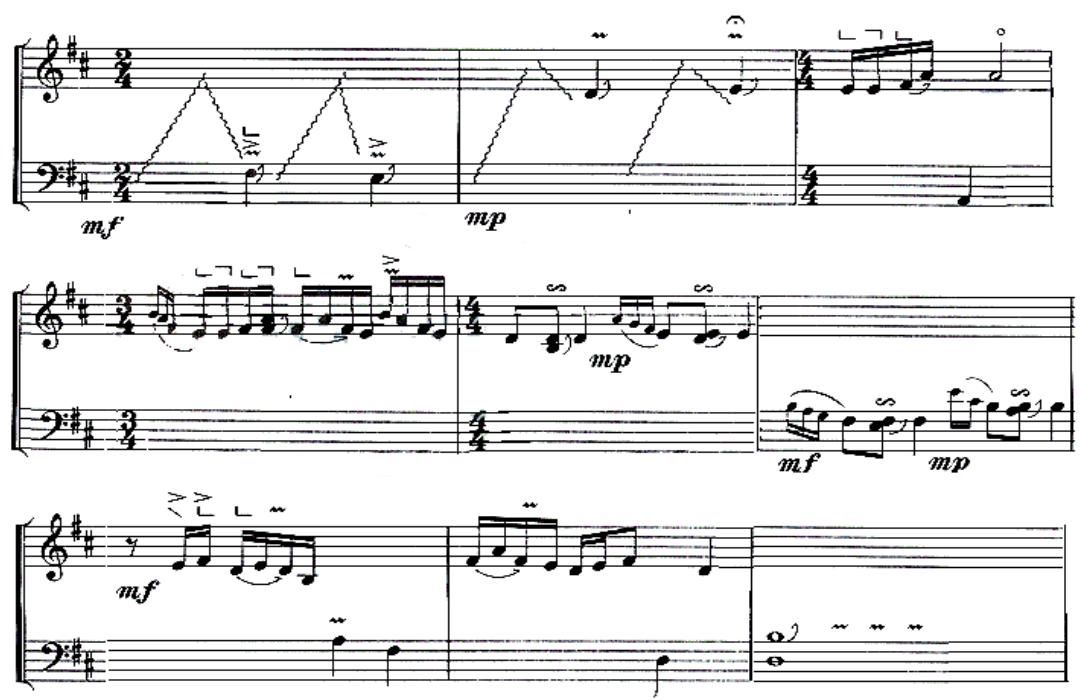

Fig. 4. Segment B, part c.

Part b1 still uses the old material of segment $b$ and the notes, rhythms and the number of sections is the same as that of segment b. The overtone technique is discarded and replaced with flatness playing, so the entire segment is raised by one octave. The composer notes here: "playing this segment near the bridge". In such way, the sound will be bright but not sharp, soft and elegant, which also highlight the quiet and noble bamboo character. As shown in "Fig. 5":
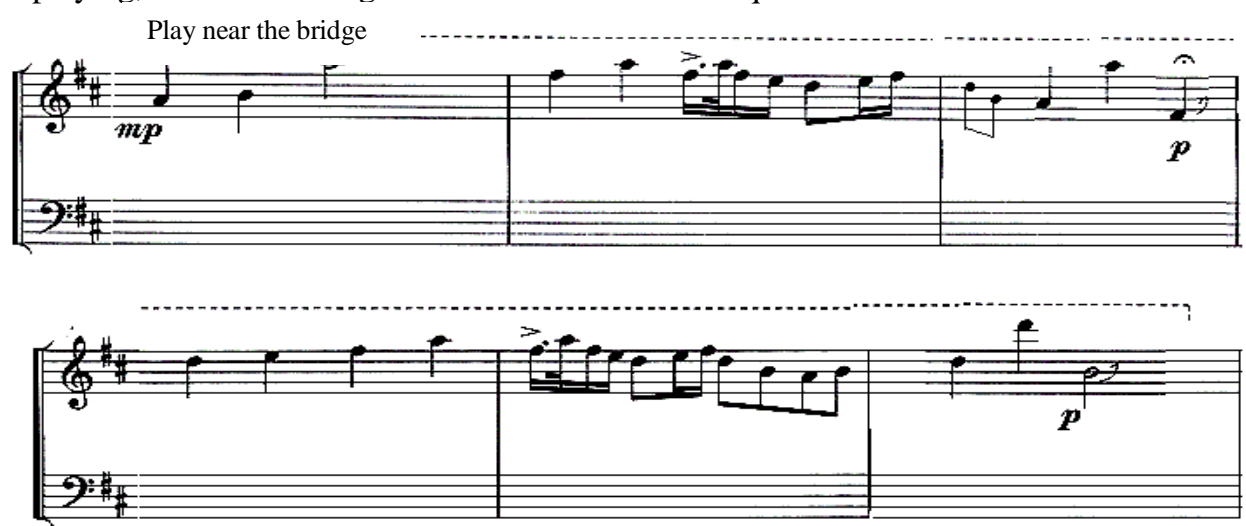

Fig. 5. Segment B, part b1.

Finally is the reappearance part, which summarizes the whole song and deepens the theme. There are two major phrases: the 6-section phrase that is the same as the introduction and the 7-section a2 phrase that uses the old material of segment $\mathrm{A}$. This is a repetitive reappearance, with the reduction and consolidation of musical terms in the middle. It not only accentuates the theme, but also maintains the concise and refined character of the music piece. As shown in "Fig 6": 


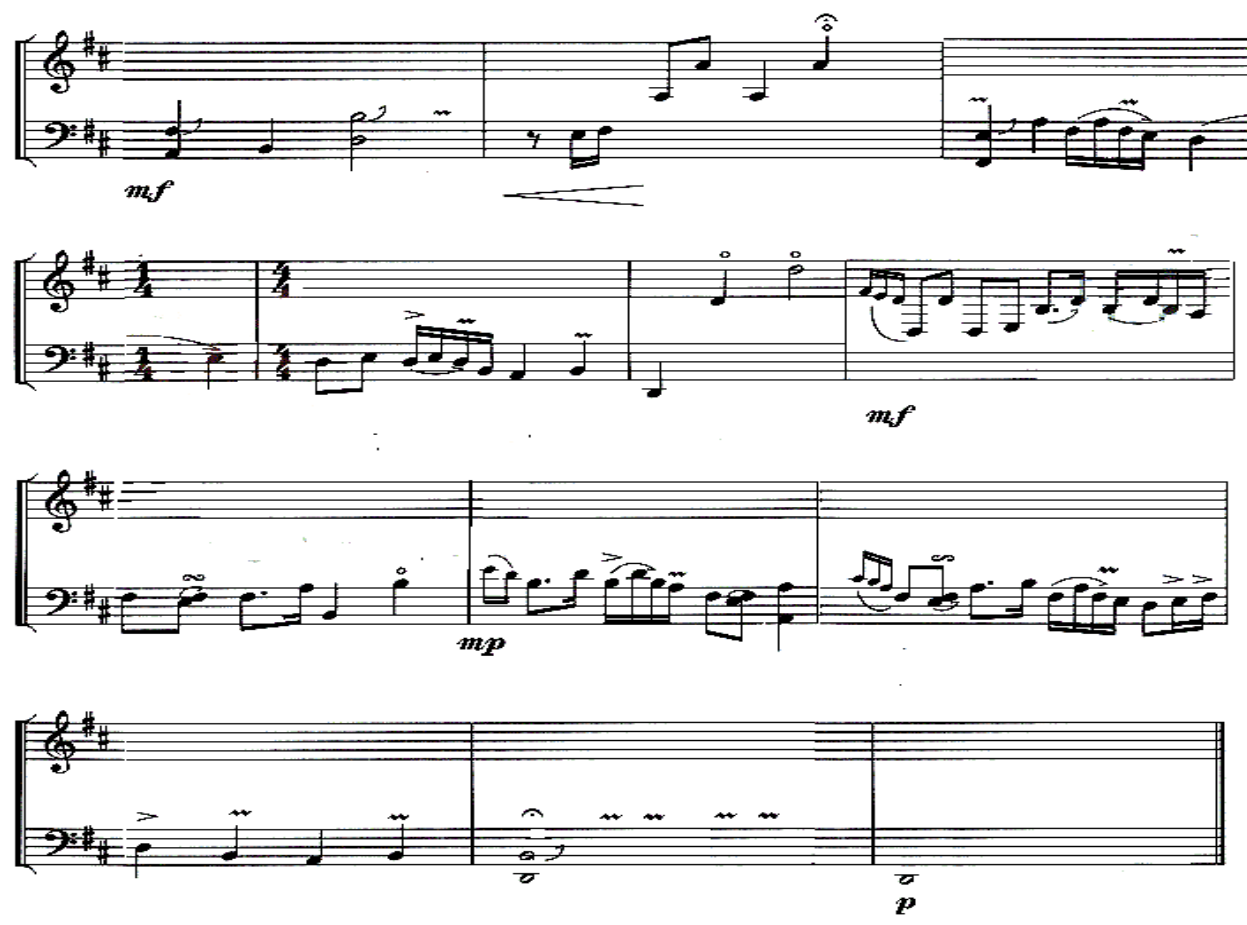

Fig. 6. Reappearance part.

In the "Bamboo Rhyme", such a phrase runs through the whole song in "Fig. 7":

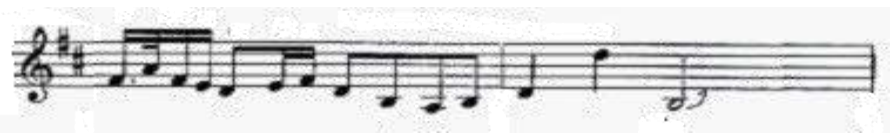

Fig. 7. Theme phrase.

This phrase intersperses in all corners of the whole song, during which many phrases are similar phrases derived from this phrase through variation and reduction. This phrase repeatedly appears several times, so it can be seen as the theme of "Bamboo Rhyme". The same melody appears many times, with a phrase repeating, which deepens the theme of music.

\section{PLAYING SKILLS AND DIFFICULTIES}

"Bamboo Rhyme" is a modern work written by traditional methods. It is composed of simple tones. This seemingly simple music is actually a great test to the player's skill. It does not have too complicated playing techniques. Only by combining the sound and the sound, integrating emotion and spirit, uniting qi and rhyme can the musical score be enriched and make "Bamboo Rhyme" shine profound artistic appeal.

\section{A. Right Hand Skills of "Bamboo Rhyme"}

The right hand skills of Zheng mainly takes the sound while traditional playing skills use the thumb, index finger and middle finger of right hand as the main force to get with the strength of the ring finger. "Bamboo rhyme" inherits the tradition, using the most basic techniques including hook, hold, wipe, pinch to take the sound. In the process of playing Zheng, we must pay attention to sound clean and avoid noise. Playing power should be concentrated in the fingertips. When touching the string, we should avoid nails making noise in the string vibrations and use lifting playing as much as possible encountering the bass zone to make the nail quickly leave the strings to avoid noise. When playing a single tone, we need to try to use paste playing techniques and put strings in advance with shoulders, arms, arms, and wrists relaxed naturally, and push out with the power of fingertips, paying attention to the balance between the intensity of sound and sound to make the sound full and penetrating. As the technique of right hand that plays the main theme, it is not difficult but attention should be paid to every detail of performance. We need to try to ensure the melody of rhythm, the rhythm, the integration of emotion and personality, and convey the spirit of performance to people.

\section{B. Left Hand Skills of "Bamboo Rhyme"}

The left hand technique of this song is the most distinctive, which is an important part of the perfect melody. The left hand technique changes the pitch by pressing the string to take sounds outside the pentatonic scale or to 
modify the proper tone. "Filling the sound with rhyme" is from here.

The left hand techniques of Zheng are basically divided into string-kneading and string-pressing, and can also be subdivided into "pressing, quivering, chanting, kneading, and sliding" and other techniques. The left hand kneading and pressing of Zheng generally has several functions: First is to distinctly and meticulously show different styles and musical images by using the changes of kneading, pointing, pressing, and sliding in close connection with the music language; Second is to play the role of changing the; third is to beautify the sound and control the sound quality; four is to play a variety of musical effects through the clever combination of left and right hand [1]. String-pressing of zheng get the required sound by changing the pitch, mainly presenting the two umlauts fa, si and the sound without fixed chord position and pitch in Zheng. "Zhu Yun" mainly uses techniques such as sliding, kneading, quivering and chanting. In addition to portamento, chanting is the most, and there is the meaning of "chanting and singing", which is the expression of inner feelings of Zheng. Among them, the prolonged sound uses the pressing method of chanting, with the left hand evenly kneading the strings up and down to create the rippling sound effects, bringing the sense of one chanting three sigh, which it is memorable.

Portamento is also used much in the song with different forms. For example in "Fig. 8":

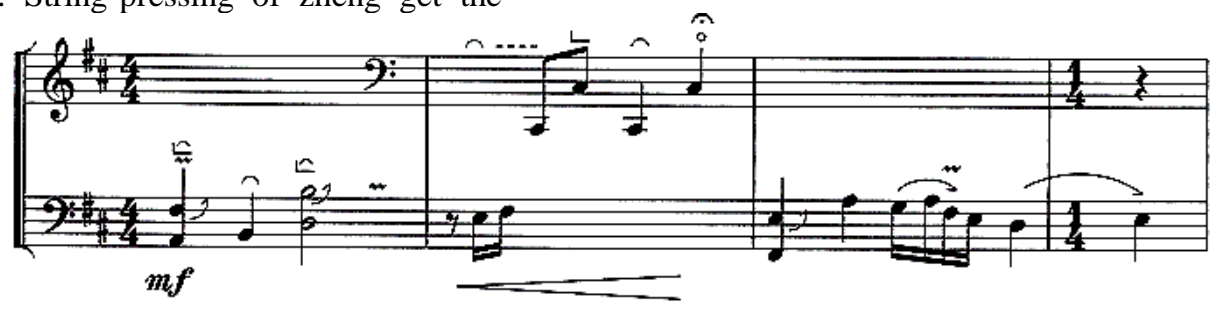

Fig. 8. Example of Left-handed technique "portamento".

The first sentence starts with the interval with portamento, and the playing power is steady. There is also summary phrase showing the meaning of completion. As the melody imitates the sound and skill of Guqin, it will lag slightly when pressed, showing the sense of space in the reverberation. On the other hand, the other kind of string press should be in strict accordance with the beat to emphasize the edges and corners. Such combination with the portamento with attached dot is like the fingers wandering between the ancient Chinese insignia with proper relax to make the phrases vivid and show the gentle charm of bamboo. The careful distinction between left hand techniques helps the performer better grasp the various pressing techniques while playing to interpret the varied color effects.

\section{Difficulties in Playing the Song}

In performance, how can we accurately express the strength, breath, music, tone, and mood is the difficulty in playing this song:

The perfect control of the intensity is the prerequisite for accurate expression of music. Changes should be made according to the analysis of the specific circumstances of the melody when playing. In the cases of relative relaxed playing in high-pitched area, we should mostly rely on the finger joints, wrist, and arm to play, so that the sound will be bright; in the bass area, we need to use the arm and body force to opponents, so the power is sure to have enough strength to play when it reaches the fingertips. The rhythm of this melody is mostly concentrated in the bass part, so attention should be paid to the rational use of the arm in playing.

Qi is the high-end form to express music, which is often said as "breathing". It is the important means to divide phrases and distinguish music level. Breathing refers to the necessary conditions for the maintenance of human life and is "qi" of aesthetics in a broad sense. Qi not only has factuality, fuzziness, transactionality, and spirituality, but also diversely unifies in the natural origin, thus deriving a number of complex concepts, such as the spirit, charm, momentum and so on [2]. Dividing the phrases with breathing is like dividing words and phrases of the language, which needs to express and apply accurately. Therefore, dividing the phrases is the basic step of breath control, so as to make the simple sound form the effect of sound broken when breath continuing. The pace of work, the degree of strength, and tone color will make breath changes differently. The emotion of "Bamboo Rhyme" is deep and calm, so the breath should be relaxed. It can be extended in the melody in the movement of music through fingering, so that we can reach the high-level realm of unity of the Zheng and player and running through of breath.

Musicality is the feeling of music, which refers to people' ability to sensitively and accurately grasp the music sound and artistic performance in the auditory experience. [3] Musicality is closely related to breath, so paying attention to the expression terms noted by composer in the score is helpful to find musicality. The tones of "Bamboo Rhyme" are concentrated in the moderate range of $\mathrm{mf}, \mathrm{mp}, \mathrm{p}$, so it is calm and soft acoustically. Paying attention to music details and expression terms, and making the appropriate musical expression will make musicality naturally enrich.

Good or bad of timbre lies in science playing method. The correct choice and application of fingering is an important factor that affects timbre, which can effectively avoid the problems of noise and thin sound. This song need to imitate the timbre of guqin, so playing by adding wiping string between sound and sound and wandering position can better express the song's vigorous and primitive meaning. 
In emotional aspect, we should pay attention to the mark of "calm, steady" in score to determine the tone of the whole song, and then analyze verbatim. Meticulous text analysis of the music should be made before playing with heart, with deeply understanding of the meaning of music; we can more accurately grasp the emotion of music.

In addition, the mental state during playing is especially important. The player should pay attention to the coordination between heart and hand to experience the direction and profound meaning of the melody so as to unify skills and emotions. In terms of artistic conception, the music can be interpreted from two aspects: first is through the impression of bamboo as a natural scene, associating with the nature and character of bamboo itself to integrate one's emotion of bamboo into music and make the emotion naturally reveal. The second is associating with the freehand brushwork of "waving brush to splash ink" in calligraphy to make the second creation of music. Calligraphy and music are different in approach but equally satisfactory in result, with close connection. Thus we can think of bamboo's rhythm and will have the feeling of gentleman when playing.

\section{The Playing ART OF "BAMBOO RHYME"}

The reason why "Bamboo Rhyme" vividly expresses the shape, temperament and spirit of bamboo is closely related to the playing art within it. It is by no means easy to touch people's heart when actually playing a piece of music with a traditional musical style. Therefore, understanding the rhythm and emotion in playing is premise for the perfect expression of music.

\section{A. Fingering and "Rhyme"}

The creation of "Bamboo Rhyme" belongs to the category of the traditional pentatonic scale. With only a few notes, it depicts the vivid image of beauty and rhyme of bamboo. Fingering is one of the key of making the playing of the song show the most perfect state. Fingering refers to the study on the fingering and the state of finger movement when playing. Only in-depth analysis of the fingering and finger movement can show the high-level state of music "rhyme." When playing, the player needs to pay attention to the state of finger touching line and use scientific methods, so that both hands can play the music in the case of relaxation. Elegant and ancient rhyme can be seen everywhere in the connection of the sound and the sound, in conversion of chanting, kneading, pressing and sliding, and in the melody of sound broken when breath continuing. Musical notes are in order in the irregular melody and rhyme transfers in the fingering. Set by breath, the rhyme and qi blend with each other, together shaping the gentleman spirit of bamboo. Players should explore the cadence in the flow of music, the intensity of the strength and speed, and body rhythm of rhythm and meter, cultivate the understanding and perception of fingering and its connotation, and express the rhyme with the contrast of light and dark lines, rigid and flexible force in the tone, to show the inner spirit of the work.

\section{B. "White Space" out of the String}

White space is a common technique in traditional Chinese painting. Drawing a few lines in Xuan paper and leaving the rest of the paper white can give the people enjoying the painting more imaginary space. For example, in "fishing alone on river" of Ma Yuan in Southern Song dynasty, there is only a boat painted, on which a fishing man is fishing. There is no water in the entire work, but people can imagine the scenery of a wide expanse of mist-covered waters after watching it. There is a similar approach in literature: "Road twists and turns but I have been unable to see you, only footprint of horse left in snow." Such statements naturally leave a deep impression of heavy snowfall. The technique of "white space" in music is reflected most vividly in "Bamboo Rhyme". There are only some simple sounds in the song, and each tone is independent from each other. However, from point to line, the left hand links the rhyme seamlessly. During the second creation, we should try our best to experience the emotion in the heart and ponder over repeatedly so as to express the music in rich layers and rhyme, grasp the sense of space existing in the music and fill the real sound with the sound in mind, so as to feel the mystery of "have" and "no".

\section{Sense of Rhythm}

Music is the art of time. It shows the extreme freedom of music in the prescribed framework of rhythm and expresses the uninhibited feeling in the heart of the player. In Chinese traditional music, the beauty of music lies in the rhythm and emotion existing in the connection between sound and sound. The scattered shape and concentrated spirit make the music have a huge sense of space and freedom. Sense of rhythm exists as the interval and duration between sounds, so after a sound is completed, we should not be hurried to play the next sound. When the rhyme of the rest sound is enough, the next sound can be made. Rhythm exists within it, so there is interest outside the strings. To understand the sense of rhythm in cadence is the main point to comprehend "Bamboo Rhyme": the musical notes extend and spread indefinitely, and the player's emotions breathe and run between the musical notes to create an ethereal artistic conception, which is also an important part that composites "bamboo rhyme".

\section{AESTHETIC IMPLICATION OF "BAMBOO RHYME"}

A deep understanding of aesthetics helps to accurately grasp the emotion in the playing style. Aesthetics studies the art or things from the perspective of aesthetic relations and analyzes from the aspects of essence, object, existence, creation, experience, consciousness, development and significance of the beauty so as to understand the beauty of art in more detail with the depth and breadth of philosophy. For "Bamboo Rhyme", the composer's target bamboo represents a kind of beauty. Bamboo, one of the four gentlemen of Chinese traditional culture, is evergreen in all seasons and stands still in cold winter. It is long, empty in middle, fearless of cold, indifferent to life and full of energy, becoming the symbol and representative of Chinese literati. It's tough and upright unyielding spirit is given new meaning 
continuously by literati of successive generations: exemplary conduct and nobility of character, neither humble nor pushy, straightforward character, which is praised by the world. The inherent integrity and virtue of bamboo is the continuation of the connotation of its cultural spirit and the bamboo culture extended last for thousands years.

Throughout the whole article, what can best represent the aesthetic connotation is the traditional Chinese culture integrated into it - "Vivid artistic conception". Aesthetics of Chinese traditional art always pay attention to meaning, love, environment, spirit, rhyme, quality, taste, wind [4]. "Vivid artistic conception" is the soul of aesthetics. The concept of "Qi" in ancient philosophy refers to the concept of primitive origin of all things in the world, which is the basis of life for all things in the world and later becomes the category of philosophy and aesthetics. The so-called "rhyme" mostly refers to the phonology of language and the rhyme of music. After the Song Dynasty, the concept of rhyme was spread to all the art fields and became the highest standard of artistic aesthetic. The concept of "vivid artistic conception" first appeared in the preface of the "Six principles of Chinese painting" of the painters Xie He during the Northern and Southern Dynasties: "What is the six-way rule? First is the vivid artistic conception. [5] Qi and rhyme depend each other, and vivid artistic conception has also become the lifelong pursuit of creation of Chinese artist. "Bamboo Rhyme" fully expresses the "physical" rhyme, and the "invisible" rhyme beyond chords. When highlighting the emotional expression, the emphasis should be placed on the "rhyme" played by the left hand to fill the gap between the simple tones. The overtones is from point to line and from virtual to true; Qi and rhyme are from inside to outside, from spirit to temperament, and from the figure to the image, lingering outside the string. It makes the whole song steady and unsophisticated, pure and sincere. The performer must continuously comprehend the artistic conception of the whole song to perceive the rhythmic vitality in the music, so that the player can perfectly interpret the playing style of the work during the second degree creation.

\section{CONCLUSION}

"Bamboo Rhyme" starts around the shape of the bamboo, using the unique skills of Zheng, to vividly express the rhythm and emotion in it. This piece of work blend emotion into scenery and depict the image of bamboo by combing feelings with the natural setting and writing both temperament and spirit from the scenery to feeling. "Bamboo Rhyme" combines Chinese traditional arts including technology, implication, philosophy and aesthetics, so only by grasping the playing style of this song and realizing the vivid atmosphere in the playing, can we fully feel the charm of Chinese folk music, and the temperament and spirit contained in it.
[2] Wang Chaoyhui. On the use of breath in pipa playing [J]. Chinese music. 2003. 1

[3] Wang Chenhui. On the sensory training in music teaching [J]. Journal of Heilongjiang College of Education 2003, 22 (4)

[4] Wang Chaoyhui. On the use of breath in pipa playing [J]. Chinese music. 2003. 1.

[5] Chen Jing. On the Qi and rhyme in the art of Zheng palying [D]. Nanjing Normal University.

\section{REFERENCES}

[1] Xu Shoucheng, Xu Lingzi. On the left hand kneading and pressing [J]. Chinese music. 1984 (3). 